\title{
Altered thermal sensitivity in facial skin in chronic whiplash-associated disorders
}

\author{
Birgitta Häggman-Henrikson ${ }^{1}$, Ewa Lampa ${ }^{1}$ and Erik Nordh ${ }^{2}$
}

There is a close functional relationship between the jaw and neck regions and it has been suggested that trigeminal sensory impairment can follow whiplash injury. Inclusion of manageable routines for valid assessment of the facial sensory capacity is thus needed for comprehensive evaluations of patients exposed to such trauma. The present study investigated facial thermal thresholds in patients with chronic whiplash-associated disorders (WADs) with both a qualitative method and quantitative sensory testing (QST). Ten women with pain and dysfunction following a whiplash injury were compared to 10 healthy age-matched women. Thermal detection thresholds were assessed by qualitative chair-side testing and by QST according to the method-of-limits. Seven test sites in the facial skin (overlying each trigeminal branch bilaterally, and the midpoint of the chin) were examined. The detection warm and cold thresholds were defined as the mean values of 10 individual thresholds. For the WAD patients, the qualitative assessment demonstrated both reduced and increased sensitivity compared to the healthy, whereas QST systematically showed significantly higher detection thresholds (i.e., decreased sensitivity) for both cold and warm stimuli. For the individuals who were assessed as having increased sensitivity in the qualitative assessment, the QST displayed either normal or higher thresholds, i.e., decreased sensitivity. The results suggest that QST is more sensitive for detecting thermal sensory disturbances in the face than a qualitative method. The impaired thermal sensitivity among the patients corroborates the notion of altered thermal detection capacity induced by WAD-related pain. International Journal of Oral Science (2013) 5, 150-154; doi:10.1038/ijos.2013.42; published online 19 July 2013

Keywords: jaw; neck; quantitative sensory testing; thermal thresholds; trigeminal; whiplash injury

\section{INTRODUCTION}

The term whiplash describes a hyperextension-flexion injury to the neck. The incidence in Sweden is about 1-2 per 1000 inhabitants, mostly from traffic injuries but also from other traumas such as falls. ${ }^{1}$ Although most individuals recover from an acute whiplash injury, ${ }^{2}$ a substantial number of individuals will develop chronic symptoms ${ }^{3}$ embraced in the term whiplash-associated disorders (WADs). The most common signs and symptoms after whiplash injury are neck pain, impaired neck movements and headaches. ${ }^{2,4}$ A range of other symptoms such as vertigo, disturbances in memory, concentration, sleep, hearing and vision functions are also reported. ${ }^{4-6}$ In addition, widespread sensory disturbances have been reported in both acute and chronic WAD. ${ }^{7-9}$

Studies in animals and humans show a close anatomical and biomechanical relationship between the jaw and neck regions, which suggests a functional linkage between the jaw-face and craniocervical sensorimotor systems. ${ }^{10}$ As jaw function rely on linked motor control of the jaw and neck motor systems, pain and dysfunction in the neck may impair jaw function. ${ }^{11}$ In chronic WAD, an association has been shown between pain and dysfunction of the neck and disturbed jaw motor function. The findings include reduced amplitude for both lower jaw and head-neck movements, disturbed coordination of jaw and head-neck movements, ${ }^{12-13}$ pain and reduced endurance during chewing. ${ }^{14-15}$

All these observations indicate an important sensorimotor integration between the jaw and neck. Although earlier studies concerning putative trigeminal sensory impairment in WAD are sparse, it has been suggested that trigeminal sensory impairment can follow whiplash injury. ${ }^{16}$ This indicates that impairment of temperature sensibility in the trigeminal skin area can be a sign of damage to the central trigeminal system. In line with this notion, it has been proposed that systematic analysis of sensory nerve function can aid in diagnosis and provide accurate follow-up. ${ }^{17} \mathrm{At}$ such evaluations, quantitative sensory testing (QST) is a widely accepted method for thin fibre evaluation of functional disturbances in afferent small fibre sensory systems and a methods-of-limits strategy is often used. High short- and long-term reproducibility and reliability show that this is a feasible method for thermal QST. ${ }^{18}$ Thus, QST is an accepted method for evaluating functional disturbances in thin afferent peripheral nerves and has been reported to be a reliable method for use also within the orofacial area. ${ }^{19}$ However, an adequate performance of QST requires good insight in psychophysical testing and hence, is not apt for widespread use as chair-side assessment in clinical routine examinations. Hence, putative alternative qualitative methods for testing have to be searched for. 


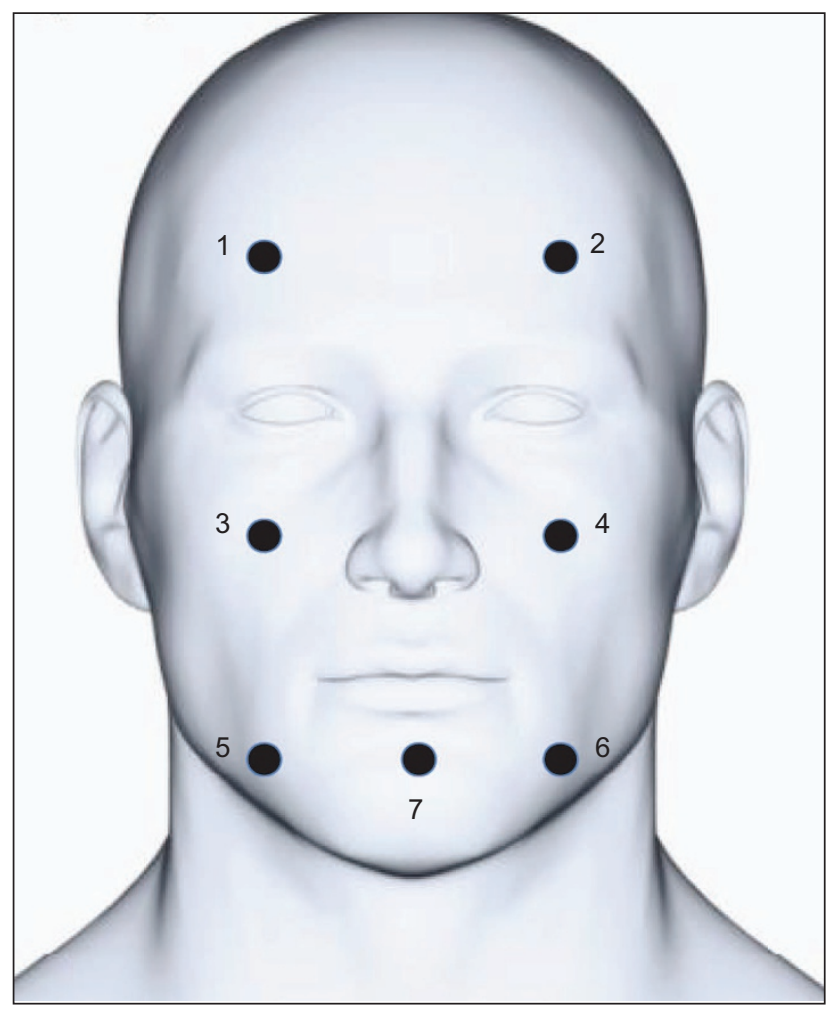

Figure 1 Seven test sites in the facial skin overlying each trigeminal branch were examined. Forehead $(1,2)$, cheek $(3,4)$, chin $(5,6)$ and the midpoint of the chin (7).

The aim of the present study was to investigate whether any signs of thermal sensory impairment could be observed in patients with chronic WAD, by analysing the thermal detection thresholds in facial skin either with a qualitative method or by QST.

\section{MATERIALS AND METHODS}

\section{Subjects}

Ten women with pain and dysfunction following a whiplash injury were compared to 10 healthy age-matched women. The WAD patients had been referred to the Department of Clinical Oral Physiology, Umeå University Hospital, Sweden, for assessment and management of jaw and neck pain and dysfunction that had developed following head-neck trauma, mostly in motor vehicle accidents, and had lasted more than 6 months. They had been diagnosed and classified by physicians as WAD class II or III according to the Quebec classification, ${ }^{2}$ in which grade I denotes neck pain, grade II neck pain with musculoskeletal signs, grade III neck pain with neurological signs and grade IV neck pain with fracture. Exclusion criteria for the healthy subjects were history of neck trauma and joint and/or general muscle diseases. All participants gave informed consent according to the World Medical Association's declaration of Helsinki. The Ethics committee of the University of Umeå approved the investigation.

\section{Test procedure}

All participants were evaluated, in random order and by two different examiners, with both a clinical qualitative assessment and a quantitative thermal test. The clinical examiner was blinded to the QST results, and vice versa. Seven test sites in the facial skin (bilaterally overlying each trigeminal branch, and also the midpoint of the chin) were examined (Figure 1). The sequences of the test sites were randomly selected for each subject. All tests were performed with the subjects comfortably seated in a reclined position in a quiet room.

For the qualitative assessment a cold (room tempered) and warm $\left(45{ }^{\circ} \mathrm{C}\right.$ ) metal spatula was applied to the facial skin. Each application was repeated three times for each modality at each test site. The subjects were asked for perception of warm and cold and were instructed to report side differences and any paradoxal sensations for example pain, hypersensitivity or discomfort.

For the QST, thermal detection thresholds (cold and warm) were assessed according to the method-of-limits (Thermotest ${ }^{\circledR}$; Somedic $\mathrm{AB}$, Hörby, Sweden) using an $18 \mathrm{~mm} \times 18 \mathrm{~mm}$ probe of Peltier element. Each location was tested with a series of 10 stimuli, starting from an adapted skin temperature of $32{ }^{\circ} \mathrm{C}$, with a warm/cold change in temperature of $\pm 0.5^{\circ} \mathrm{C} \cdot \mathrm{s}^{-1}$ and a return speed of $1{ }^{\circ} \mathrm{C} \cdot \mathrm{s}^{-1}$. Subjects were instructed to immediately report the perception of a hot or cold sensation by pressing a handheld electrical switch. This caused a readout of the prevailing probe temperature and returned the temperature back to the start temperature of $32{ }^{\circ} \mathrm{C}$. Between each temperature stimuli, there was a randomly selected interval of $3-5 \mathrm{~s}$. The thresholds (cold and warm) for each location were defined as the means of the recorded ten individual thresholds in the sequence. Details of experimental set-up have been given previously. ${ }^{18}$

\section{Statistical analysis}

Median, minimum and maximum values were used for descriptive statistics. Differences between test locations and between the WAD group and the healthy group were tested with two-tailed Wilcoxon matched-pairs test and Mann-Whitney $U$ test, respectively, with a probability level of 0.05 .

\section{RESULTS}

\section{Qualitative assessment}

The clinical assessment demonstrated both reduced and increased thermal sensitivity in the WAD group, whereas almost no sensory disturbances were found for healthy (Table 1). There were no significant differences in median values between the groups. Some WAD individuals also reported paradoxical sensations such as spreading of sensation (subjects Nos. 5 and 9), numbness (No. 5), a slight burning sensation (No. 7), or a combination of numbness and burning (No. 9). Many of the WAD subjects also reported a lingering sensation after the stimuli (Nos. 4, 5, 7, 8 and 9).

\section{QST (Thermotest)}

There were no significant intra-individual side-to-side differences for the whiplash and healthy groups. Therefore, the data from the left and right sides were pooled, and mean values for the bilateral sites calculated for each individual.

There was large inter-individual variability in thermal detection thresholds. Generally, the forehead location showed higher variability compared to other sites, and when tested for heat, also a higher temperature threshold. The relative detection threshold values were generally lower for cold stimuli than for warm stimuli.

Compared to healthy, the WAD patients showed higher detection thresholds (i.e., reduced sensitivity) for both cold and warm stimuli (Figure 2). These differences were significant for the cheek and chin sites, but not for the forehead.

For the WAD individuals who were assessed as having increased sensitivity in the qualitative examination, the QST displayed either normal or higher thresholds, i.e., reduced sensitivity. In the WAD 
Table 1 Qualitative assessment of thermal thresholds to cold and warm for healthy $(n=10)$ and WAD ( $n=10)$ subjects for the different test sites. Order of subjects arranged in relation to sensory disturbances

\begin{tabular}{|c|c|c|c|c|c|c|c|c|c|c|c|c|c|c|}
\hline \multirow[b]{2}{*}{ Subjects } & \multicolumn{7}{|c|}{ Cold } & \multicolumn{7}{|c|}{ Warm } \\
\hline & Left & Right & Left & Right & Left & Right & Middle & Left & Right & Left & Right & Left & Right & Middle \\
\hline \multicolumn{15}{|l|}{ Healthy } \\
\hline \multicolumn{15}{|l|}{1} \\
\hline \multicolumn{15}{|l|}{2} \\
\hline \multicolumn{15}{|l|}{3} \\
\hline \multicolumn{15}{|l|}{5} \\
\hline \multicolumn{15}{|l|}{6} \\
\hline \multicolumn{15}{|l|}{7} \\
\hline \multicolumn{15}{|l|}{8} \\
\hline \multicolumn{15}{|l|}{9} \\
\hline 10 & & & & & & & & & & $(+)$ & $(+)$ & & & \\
\hline 5 & & - & & - & & - & - & & - & & - & & - & \\
\hline 6 & & & & & & & + & & & & & & & + \\
\hline 7 & & $(+)$ & + & & & & & & & & & & + & \\
\hline 8 & & + & & + & & + & & & + & & + & & + & \\
\hline 9 & & + & & + & & ++ & & -- & -- & & + & & + & \\
\hline 10 & & ++ & & ++ & & ++ & & & ++ & & ++ & & & \\
\hline
\end{tabular}

WAD, whiplash-associated disorder.

$(-)$ : slightly reduced temperature sensitivity; $(+)$ : slightly increased temperature sensitivity; -: reduced temperature sensitivity; +: increased temperature sensitivity; - - : absence of temperature sensation; ++: markedly increased temperature sensitivity.
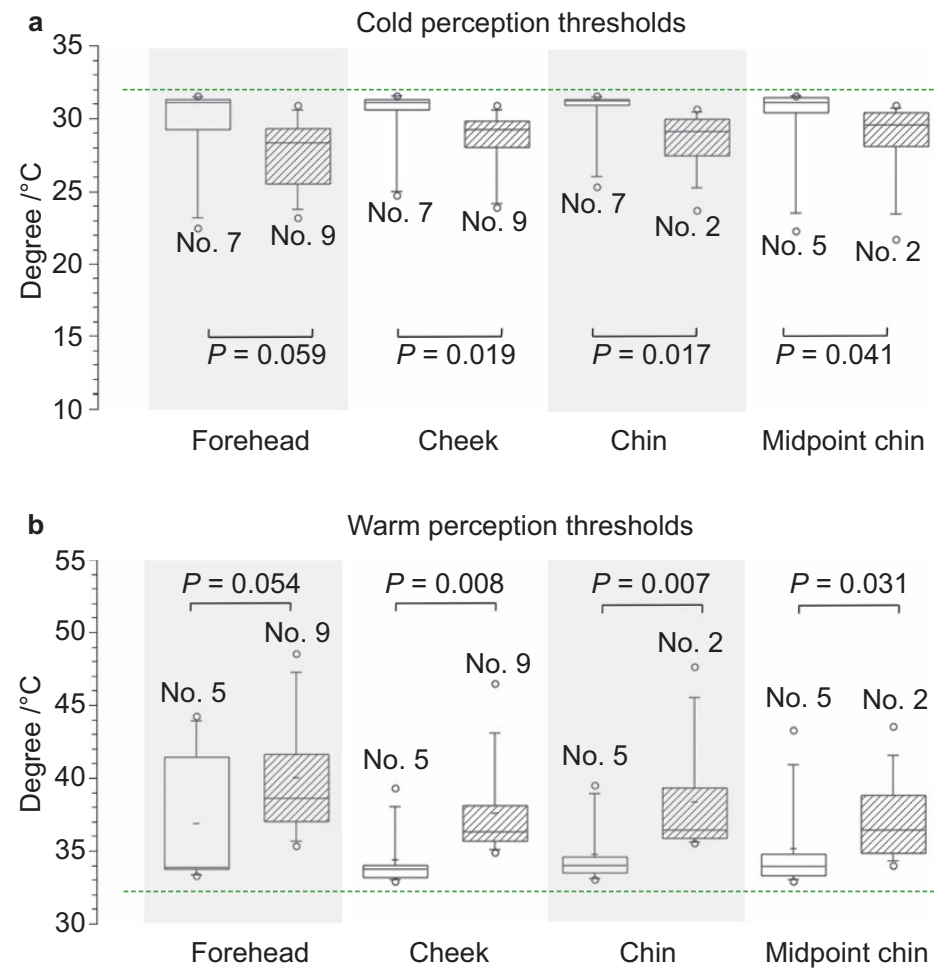

Figure 2 Thermal cold and warm detection thresholds at the different test sites, for healthy (unfilled) and WAD (hatched) subjects in relation to the baseline temperature of $32{ }^{\circ} \mathbf{C}$. (a) Cold perception thresholds. (b) Warm perception thresholds. Numbers besides outliers refer to subjects' numbers as given in Table 1. WAD, whiplash-associated disorder. 
group, the QST triggered a wide range of paradoxical sensations such as stabbing, pain, numbness and tingling (No. 9), cold sensation when tested for heat (No. 4) and spread of sensation (No. 8).

\section{DISCUSSION}

The present study investigated thermal detection thresholds in facial skin in chronic WAD patients and healthy with both a qualitative method and QST. The main findings were that for the WAD patients, the qualitative assessment demonstrated both reduced and increased sensitivity, whereas the QST showed normal or higher thermal detection thresholds (i.e., decreased sensitivity) for both cold and warm stimuli.

The data collection for the present study was carried out before new guidelines for QST assessment for orofacial pain were published. ${ }^{20}$ However, the methodology we used both for the clinical chair-side assessment and the QST, are in line with these recommendations for assessment of thermal thresholds in facial skin. It is generally accepted that a qualitative examination by a clinical chair-side assessment should be used as a screening tool rather than to determine the degree of sensory disturbances. At group level, the present study did not show any differences between healthy and patients with the qualitative assessment, although both increased and decreased temperature sensitivity was observed for individual patients.

The quantitatively assessed absolute values of thermal detection thresholds in the present study were generally higher than previously reported. Partly, this may be caused by differences in probe size between studies. For the QST, we used a small probe (less than $4 \mathrm{~cm}$ area), which is in accordance with the guidelines, ${ }^{20}$ whereas previous investigations in patients with WAD were done with a larger probe. ${ }^{16,21} \mathrm{~A}$ smaller sized probe has been deemed more suitable for the trigeminal area in order to assess the individual branches of the trigeminal nerve, as well as to avoid spatial summation. Also age may be a cause for differences as previously a lower age of subjects was reported, ${ }^{19}$ and an increase in thermal perception thresholds in facial skin with age has been demonstrated. $^{22-23}$ The use of an extra-trigeminal reference site is also recommended by the guidelines, ${ }^{20}$ which was not used neither in our, nor in previous studies of WAD patients. ${ }^{16,21}$ Such a rationale is therefore recommended for future studies, as trigeminal sensory disturbances in WAD patients indeed may be part of a widespread phenomenon, in analogy with jaw pain in WAD being part of a widespread pain syndrome. ${ }^{24}$ In patients with localized jaw pain and dysfunction, temporomandibular disorders, thermal pain thresholds, but not detection thresholds, were reported to differ from healthy subjects in trigeminal and extra-trigeminal regions. ${ }^{25}$

With regard to the QST, there was generally large inter-individual variability in temperature thresholds, and more so in the patient group and for the first trigeminal branch. This is in accordance with previous reports both in WAD patients, ${ }^{16}$ and in healthy subjects. ${ }^{26}$ Generally, this variability was more pronounced for heat detection thresholds, which is also in line with the earlier reports. The higher variability in thresholds for the first trigeminal branch may be related to the lower density of thermal receptors in this area of facial skin, compared to the higher density nearer the orofacial cavity. In analogy, the higher temperature thresholds observed at the forehead in the present study, more evident when tested for heat, would corroborate this notion.

At QST, the WAD patients generally had higher thresholds to both cold and warm stimuli, compared to the gender- and age-matched group with healthy individuals. The generally decreased thermal sensitivity in these patients indicates interference to thermal detection capacity induced by pain. An important methodological aspect to be kept in mind when considering the QST findings is that the test is of psychophysical nature. Thus, the result is affected by the functional state in all of the constituting components, from the peripheral receptors over the afferent fibres and the ascending pathways to the central evaluating processes. Although the increased sensitivity found at the qualitative assessment could reflect a tendency for thermal receptors to activate pain pathways or for a sensitization of nociceptors, the increased sensitivity and the paradoxical sensations might equally well reflect a tendency for increased central sensitisation, parallelling that which can be seen in conditions with spinal segmental upregulation. However, the fact that most pathological findings were bilateral, indicates that the pathophysiological mechanism is less likely to be a peripherally located injury, but rather corresponds to functional changes located in more centrally located structures than the brainstem or the upper spinal cord.

Interestingly, in WAD patients, thermal thresholds are increased, while hypersensitivity to pain is present. It has been suggested that the ongoing nociception may induce central inhibitory mechanisms, which could reduce the thermal sensitivity. ${ }^{27}$ Also, a relationship between sensory disturbances and symptoms related to the central nervous system (face pain and numbness, dizziness, sensitivity to light and/or sound, memory and concentration problems) has been reported in WAD patients. ${ }^{21}$

In the present study, all WAD patients showed sensory disturbances and presented with jaw pain. Jaw pain can be part of the overall spectrum of symptoms in $\mathrm{WAD}^{28-30}$ and some patients also report pain during eating and chewing. ${ }^{14-15}$ Furthermore, it has also been reported in chronic WAD that this jaw pain can be frequent and combined with symptoms of numbness in the jaw-face region, ${ }^{31}$ which might indicate a disturbed trigeminal sensory nerve function. This also corroborates previous findings of a correlation between increased trigeminal thermal thresholds and pain and numbness in the face, ${ }^{21}$ although no correlation to the intensity of neck pain was found. Furthermore, results from experimental studies support the existence of intersegmental nociceptive connections between the cervical spine and the trigeminal nuclei ${ }^{32}$ and there is support in experimental ${ }^{33}$ as well as clinical ${ }^{34}$ studies for overlapping spread and referral of muscle pain between the cervical and trigeminal regions.

\section{CONCLUSIONS}

The finding of increased thresholds in facial skin following whiplash injury is concordant with both the findings of jaw pain and disturbed jaw function after neck injury, as well as with the reports of neural connections between the central sensory systems of the two body regions. The present findings also further underline the sensory-motor integration between the jaw and neck regions. Finally, the results indicate that simplified clinical rationales for testing of the facial thermal discrimination capacity, and hence, the state of important components of the trigeminal small fibre modalities, may not be sufficient for accurate descriptions of the sensory function in patients suffering from head-neck pain of different origins.

\section{ACKNOWLEDGEMENTS}

This work was supported by the Department of Odontology and the Faculty of Medicine, Umeå University, the Folksam Research Foundation and grants from Västerbotten County Council (TUA).

1 Björnstig U, Hildingsson C, Toolanen G. Soft-tissue injury of the neck in a hospital based material. Scand J Soc Med 1990; 18(4): 263-267. 
2 Spitzer W, Skovron M, Salmi L et al. Scientific monograph of the Quebec task force on Whiplash-Associated Disorders: redefining "whiplash" and its management. Spine 1995; 20(8 Suppl): 1S-73S.

3 Freeman MD, Croft AC, Rossignol AM et al. A review and methodologic critique of the literature refuting whiplash syndrome. Spine (Phila Pa 1976) 1999; 24(1): 86-96.

4 Sterner Y, Gerdle B. Acute and chronic whiplash disorders-a review. J Rehabil Med 2004; 36(5): 193-209.

5 Guez M, Brännstrom R, Nyberg L et al. Neuropsychological functioning and MMPI-2 profiles in chronic neck pain: a comparison of whiplash and non-traumatic groups. J Clin Exp Neuropsychol 2005; 27(2): 151-163.

6 Stålnacke BM. Relationship between symptoms and psychological factors five years after whiplash injury. J Rehabil Med 2009; 41(5): 353-359.

7 Chien A, Eliav E, Sterling M. Hypoaesthesia occurs with sensory hypersensitivity in chronic whiplash-further evidence of a neuropathic condition. Man Ther 2009; 14(2): 138-146

8 Chien A, Eliav E, Sterling M. The development of sensory hypoesthesia after whiplash injury. Clin J Pain 2010; 26(8): 722-728.

9 Sterling M, Jull G, Vicenzino B et al. Sensory hypersensitivity occurs soon after whiplash injury and is associated with poor recovery. Pain 2003; 104(3): 509-517.

10 Eriksson PO, Häggman-Henrikson B, Nordh E et al. Co-ordinated mandibular and head-neck movements during rhythmic jaw activities in man. J Dent Res 2000; 79(6): 1378-1384.

11 Eriksson PO, Häggman-Henrikson B, Hamayun Z. Jaw-neck dysfunction in whiplashassociated disorders. Arch Oral Biol 2007; 52(4): 404-408.

12 Eriksson PO, Zafar H, Häggman-Henrikson B. Deranged jaw-neck motor control in whiplash-associated disorders. Eur J Oral Sci 2004; 112(1): 25-32.

13 Häggman-Henrikson B, Zafar H, Eriksson PO. Disturbed jaw behavior in whiplashassociated disorders during rhythmic jaw movements. J Dent Res 2002; 81(11): 747-751.

14 Grönqvist J, Häggman-Henrikson B, Eriksson PO. Impaired jaw function and eating difficulties in whiplash-associated disorders. Swed Dent J 2008; 32(4): 171-177.

15 Häggman-Henrikson B, Österlund C, Eriksson PO. Endurance during chewing in whiplash-associated disorders and TMD. J Dent Res 2004; 83(12): 946-950.

16 Knibestöl M, Hildingsson C, Toolanen G. Trigeminal sensory impairment after softtissue injury of the cervical spine. A quantitative evaluation of cutaneous thresholds for vibration and temperature. Acta Neurol Scand 1990; 82(4): 271-276.

17 Eliav E, Gracely RH, Nahlieli 0 et al. Quantitative sensory testing in trigeminal nerve damage assessment. J Orofac Pain 2004; 18(4): 339-344.

18 Heldestad V, Linder J, Sellersjo L et al. Reproducibility and influence of test modality order on thermal perception and thermal pain thresholds in quantitative sensory testing. Clin Neurophysiol 2010; 121(11): 1878-1885.

19 Pigg M, Baad-Hansen L, Svensson $\mathrm{P}$ et al. Reliability of intraoral quantitative sensory testing (QST). Pain 2010; 148(2): 220-226.
20 Svensson P, Baad-Hansen L, Pigg M et al. Guidelines and recommendations for assessment of somatosensory function in oro-facial pain conditions-a taskforce report. J Oral Rehabil 2011; 38(5): 366-394.

21 Sterner Y, Toolanen G, Knibestöl M et al. Prospective study of trigeminal sensibility after whiplash trauma. J Spinal Disord 2001; 14(6): 479-486.

22 Yekta SS, Smeets R, Stein JM et al. Assessment of trigeminal nerve functions by quantitative sensory testing in patients and healthy volunteers. J Oral Maxillofac Surg 2010; 68(10): 2437-2451

23 Kaplan I, Levin T, Papoiu AD et al. Thermal sensory and pain thresholds in the tongue and chin change with age, but are not altered in burning mouth syndrome. Skin Res Technol 2011; 17(2): 196-200.

24 Visscher C, Hofman N, Mes C et al. Is temporomandibular pain in chronic whiplashassociated disorders part of a more widespread pain syndrome? Clin J Pain 2005; 21(4): 353-357.

25 Fernandez-de-las-Penas C, Galan-del-Rio F, Ortega-Santiago R et al. Bilateral therma hyperalgesia in trigeminal and extra-trigeminal regions in patients with myofascial temporomandibular disorders. Exp Brain Res 2010; 202(1): 171-179.

26 Becser N, Sand T, Zwart JA. Reliability of cephalic thermal thresholds in healthy subjects. Cephalalgia 1998; 18(8): 574-582.

27 Chien A, Sterling M. Sensory hypoaesthesia is a feature of chronic whiplash but not chronic idiopathic neck pain. Man Ther 2010; 15(1): 48-53.

28 Carroll LJ, Ferrari R, Cassidy JD. Reduced or painful jaw movements after collisionrelated injuiries: a population-based study. J Am Dent Assoc 2007; 138(1): 86-93.

29 Klobas L, Tegelberg A, Axelsson S. Symptoms and signs of temporomandibular disorders in individuals with chronic whiplash-associated disorders. Swed Dent $J$ 2004; 28(1): 29-36.

30 Sale $\mathrm{H}$, Isberg A. Delayed temporomandibular joint pain and dysfunction induced by whiplash trauma: a controlled prospective study. J Am Dent Assoc 2007; 138(8): 1084-1091.

31 Häggman-Henrikson B, Grönqvist J, Eriksson PO. Frequent jaw-face pain in chronic Whiplash-Associated Disorders. Swed Dent J 2011; 35(3): 123-131.

32 Svensson P, Wang K, Sessle BJ et al. Associations between pain and neuromuscular activity in the human jaw and neck muscles. Pain 2004; 109(3): 225-232.

33 Hellström F, Thunberg J, Bergenheim M et al. Elevated intramuscular concentration of bradykinin in jaw muscle increases the fusimotor drive to neck muscles in the cat. $J$ Dent Res 2000; 79(10): 1815-1822.

34 Wiesinger $\mathrm{B}$, Malker $\mathrm{H}$, Englund $\mathrm{E}$ et al. Does a dose-response relation exist between spinal pain and temporomandibular disorders? BMC Musculoskelet Disord 2009; 10 28.

(9) This work is licensed under a Creative Commons Attribution-NonCommercial-NoDerivative Works 3.0 Unported License. To view a copy of this license, visit http:// creativecommons.org/licenses/by-nc-nd/3.0 